\title{
Cono cervical con radiocirugía: alternativa en diagnóstico y tratamiento de neoplasia intraepitelial del cervix
}

\author{
Néstor H. Amorocho*; Martín E. Guardiola**
}

\begin{abstract}
RESUMEN: Se revisa el manejo de la neoplasia intraepitelial del cervix (NIC) con indicación de cono cervical diagnóstico o de tratamiento efectuado con radiocirugía, evaluando los resultados en 35 pacientes en el Hospital San Juan de Dios de Cali, en el departamento de Ginecología, obteniéndose resultados a corto plazo de mayor beneficio para la paciente tanto en el costo, como recuperación, complicaciones y resultados del tratamiento. El 57,1\% de los conos fue con fin diagnóstico y el $42.9 \%$ con fin de tratamiento. De los conos diagnósticos, el $75 \%$ resultó terapéutico y el $25 \%$ no terapéutico. De los conos de tratamiento, el $100 \%$ fue terapéutico. Se demuestra la utilidad de la radiocirugía en el manejo de NIC con fines diagnósticos o de tratamiento.
\end{abstract}

PALABRAS CLAVES: NIC, radiocirugía, cono diagnóstico, cono de tratamiento, cono terapéutico, cono no terapéutico, Lletz.

SUMMARY: A review of the management of the cervix intraepitelial neoplasia (CIN) with indication of the cervical cone diagnosis or radiosurgery treatment, evaluating the results in 35 patients in the San Juan de Dios Hospital At Cali, department of gynecology, obtaining short term benefits for the patients both in cost as well as in recuperation and treatment results.

$57,1 \%$ of the cones were addressed to diagnosis and $42,9 \%$ to treatment. $75 \%$ of the diagnosis cones resulted terapeutical and $25 \%$ unterapeutical.

$100 \%$ of the treatment cones were terapeutical. The utility of radio-surgery in handling CIN either for diagnosis or treatment is demonstrated.

KEY WORDS: CIN, Radio-surgery, diagnosis cone, treatment cone, terapeutical cone, non-terapeutical cone, Lletz.

\section{Introducción}

El manejo de la paciente con citología alterada es evaluado con colposcopia y biopsia dirigida bajo colposcopia, con el fin de precisar el diagnóstico y posterior tratamiento. Cuando no existe correlación entre la citología, colposcopia y biopsia, se requiere de estudio histológico mayor por medio del cono. Las indicaciones tenidas en cuenta para efectuar el cono son entre otras la colposcopia no satisfactoria, discordancia entre la citología, colposcopia y la biopsia, citología intracervical positiva, lesión exocervical no candidata a crioterapia dado su tamaño y el compromiso glandular (19-20).

Las definiciones tenidas en cuenta son:

- Cono diagnóstico: cuando se encuentra discordancia entre la citología y/o colposcopia y/o biopsia, se recurre al cono con el fin de establecer un diagnóstico.

- Cono de tratamiento: Cuando hay correlación entre la citología, la colposcopia y la biopsia y se observa totalmente la lesión entonces se efectúa un cono con fin de tratamiento.

Hospital de San Juan De Dios - Cali.

* Jefe Departamento de Ginecología. Docente Adjunto - Univalle

** Ginecólogo Adscrito.
- Cono terapéutico: Cuando el borde de sección quirúrgico es informado libre de lesión o alteración celular, se concluye que se hizo tratamiento.

- Cono no terapéutico: cuando el borde de sección es informado con lesión parcial o totalmente.

Dentro de las distintas alternativas para efectuar el cono se encuentra la radiocirugía, la cual se efectúa con un electrodo de radiofrecuencia en forma de asa, para obtener el tejido del cervix y endocervix deseado (Lletz: Large Loop Excisión Transformation Zone).

\section{Material y métodos}

Se revisan los resultados de 35 pacientes a quienes se les practicó cono cervical con el asa de radiocirugía en el Hospital San Juan de Dios de la ciudad de Cali en el Servicio de Ginecología entre mayo de 1992 y enero de 1993.

El cono con asa se efectuó en el Servicio de Consulta Externa de Ginecología con la paciente en posición de litotomía, utilizando anestesia local con lidocaína al $1 \%$ a nivel cervical.

Se verifica la colposcopia y se efectúa tinción con Lugol para identificar la lesión e iniciar el procedimiento con el asa para corte efectuando el cono, según el tamaño de la lesión. Posteriormente se efectúa un segundo corte para obtener tejido endocervical con una asa pequeña. Se efec- 
túa coagulación del lecho cervical con radiofrecuencia y percloruro de hierro adicional en caso necesario.

El tiempo total del procedimiento es de 10 minutos aproximadamente, se encontró como complicación en 2 pacientes un sangramiento el cual requirió de punto de Catgut Crómico en una de ellas para hacer hemostasia.

Las edades de las pacientes oscilan entre 20 y 74 años, la paridad oscila entre 00 y 10 partos. Gráficas 1-2.

\section{Gráfico 1} EDADES

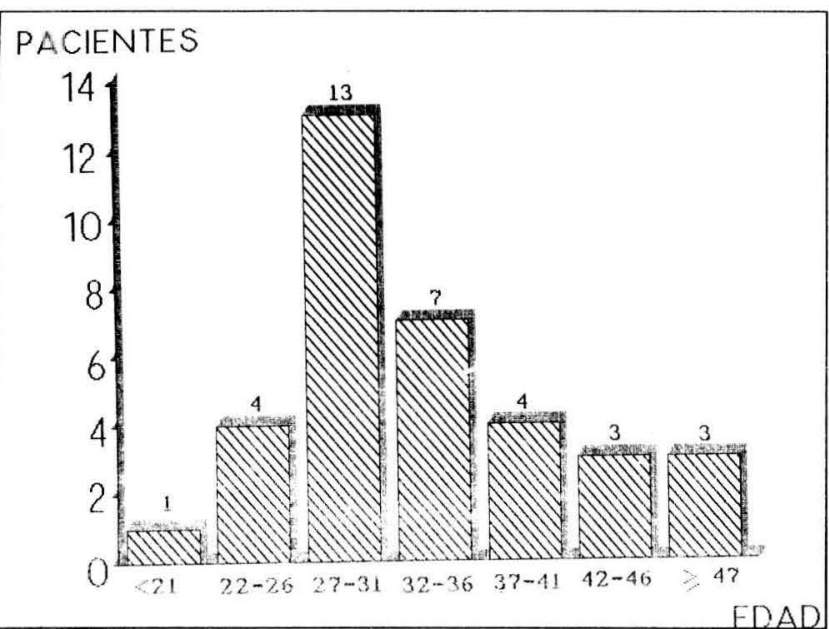

Gráfico 2

PARIDAD

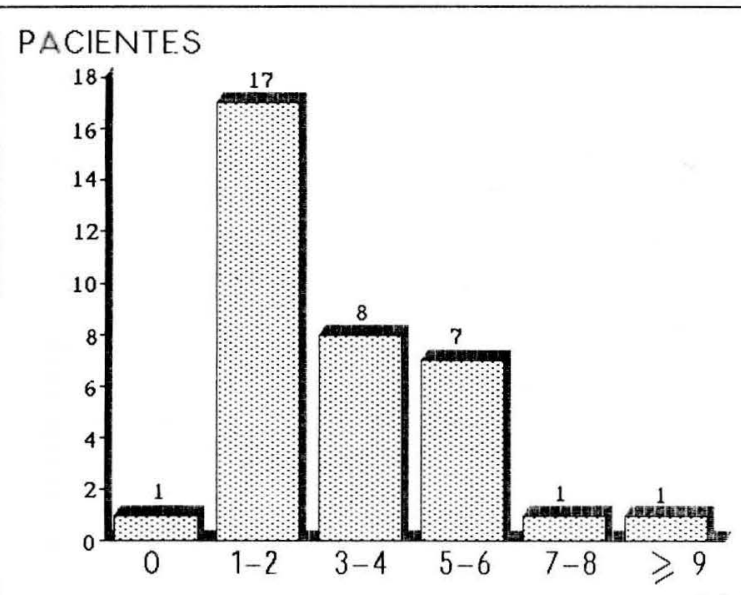

PARTOS

Las pacientes solicitaron ser atendidas en este programa en el Centro de Patología Cervico-Uterina por presentar citología cervical alterada. Para el estudio y tratamiento de las pacientes se siguieron las normas técnicas sobre patología cervical del Programa del Instituto Nacional de Cancerología sobre Detección Precoz del Cáncer Cervical.

\section{Resultados}

La citología fue informada sugerente de NIC I en $45,7 \%$ (16/35), sugerente de NIC II en $25.7 \%$ (9/35), sugerente de
NIC III en $20 \%(7 / 35)$ y sugerente de Invasor en $8,6 \%$ 35). Cuadro 1.

Cuadro 1

DIAGNOSTICO CITOLOGICO

\begin{tabular}{|c|l|}
\hline CITOLOGIA & SUGERENTE \\
\hline \multirow{2}{*}{ III } & $45.7 \%$ NIC I: $16-35$ \\
\cline { 2 - 2 } & $25.7 \%$ NIC II: $9-35$ \\
\hline \multirow{2}{*}{ IV } & $20 \%$ NIC III: $7-35$ \\
\hline V & $8.6 \%$ INVASOR: $3-35$ \\
\hline
\end{tabular}

La colposcopia fue satisfactoria en $82.8 \%$ (29/35) pacientes con visualización total de la lesión en 60\% (21/35). Las imágenes colposcópicas fueron sugerentes de NIC I en $34,3 \%$ (12/35), NIC II en $11,4 \%$ (4/35), NIC III en $40 \%$ (14/ $35)$ e inflamatorio en $14,3 \%(5 / 35)$. Cuadro 2.

Cuadro 2

DIAGNOSTICO COLPOSCOPICO

\begin{tabular}{|c|c|c|}
\hline COLPOSCOPIA & \multicolumn{2}{|c|}{ DX COLPOSCOPICO } \\
\hline \multirow{4}{*}{$\begin{array}{c}\text { SATISFACTORIA: } 82.8 \% \\
(29-35)\end{array}$} & NIC: I & $: 9$ \\
\hline & NIC: II & $: 3$ \\
\hline & NIC: III & $: 12$ \\
\hline & Inflamatorio: & $: 5$ \\
\hline \multirow{4}{*}{$\begin{array}{l}\text { NO SATISFACTORIA: } \\
17.2 \%(6-35)\end{array}$} & NIC: I & \\
\hline & NIC: II & \\
\hline & NIC: III & $: 2$ \\
\hline & Inflamatorio: & $: 0$ \\
\hline
\end{tabular}

La biopsia cervical fue procesada y leída en el departamento de patología del Hospital San Juan de Dios encontrándose biopsia concordante con la colposcopia en $71,4 \%$ $(25 / 35)$, diferencia entre la biopsia y el diagnóstico colposcópico en un grado superior en $11,4 \%(4 / 35)$ y diferencia entre el diagnóstico colposcópico y la biopsia en mayor o igual a 2 grados en $17,2 \%$ (6/35). Cuadro 3.

La patología fue enviada al servicio de patología del Hospital San Juan de Dios encontrándose igual resultado del cono al reportado en la biopsia en $82,8 \%$ (29/35), diferencia en un grado menor $5,6 \%(2 / 35)$, en un grado mayor $2,8 \%(1 / 35)$ y mayor en 2 grados en $8.6 \%(3 / 35)$. Cuadro 4 
Cuadro 3

BIOPSIA V/S COLPOSCOPIA

CONCORDANTE A COLPOSCOPIA

$71.4 \%$

( 25-35)

NO CONCORDANTE A COLPOSCOPIA : $28.6 \%$ $(10-35)$

\begin{tabular}{l} 
- Un grado superior : $11.4 \%(4-35)$ \\
\hline $\begin{array}{l}\text { Mayor o igual a } \\
\text { dos grados }\end{array}$
\end{tabular}

Cuadro 4

CONO V/S BIOPSIA

IGUAL $82.8 \%$

(29-35)

DIFERENTE $17.0 \%$

$$
(6-35)
$$

- Un grado mayor: $2.8 \%(1-35)$

- Un grado menor: $5.6 \%(2-35)$

- Mayor 2 grados: 8.6\% (3-35)

De los conos efectuados, el $57.1 \%$ (20/35) fue con fin diagnóstico y el $42,9 \%(15 / 35)$ con fin de tratamiento. Cuadro 5.

Cuadro 5

FINALIDAD DEL CONO

\section{DIAGNOSTICO: $57.1 \%$}

$$
\text { (20-35) }
$$

* Colposcopia no satisfactoria: $6 / 20$

* Lesión no visible totalmente: 10/20

* No correlación citologíacolposcopia - biopsia

TRATAMIENTO: $42.9 \%$

$$
(15-35)
$$

De los conos diagnósticos, el 75\% (15/20) salió terapéutico y el 25\% (5/20) resultó no terapéutico. En las pacientes con cono no terapéutico, se efectuó histerectomía abdominal ampliada a una paciente, encontrándose en la patología ausencia de lesión en el cervix remanente; en otra paciente con cono no terapéutico se decidió no efectuarle cirugía dado que cursaba un embarazo del primer trimestre. Las 3 pacientes restantes con cono no terapéutico se han seguido con controles citológicos y colposcópicos sin encontrarse alteración de estos. De los conos con fin de tratamiento, el 100\% resultó terapéutico. Cuadro 6.

Cuadro 6

RESULTADO DEL CONO

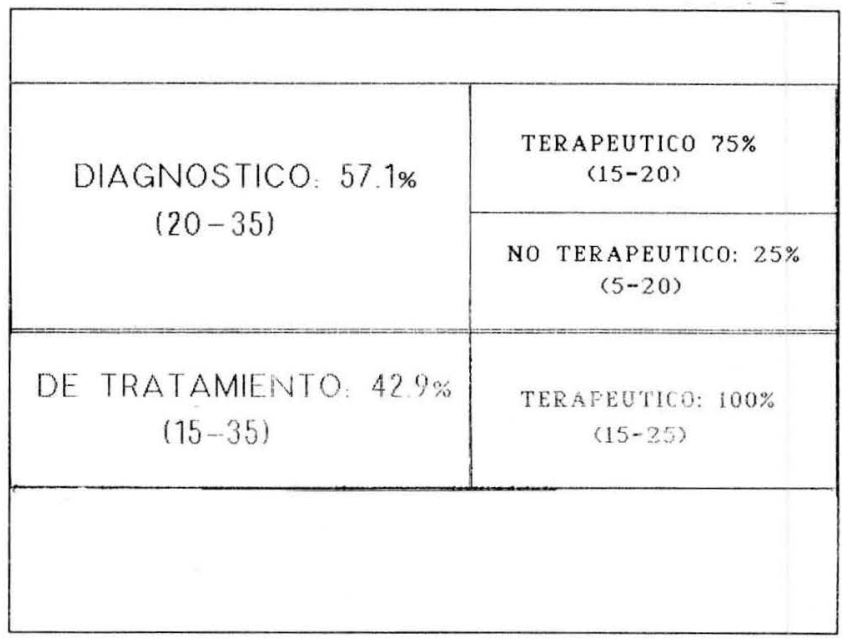

El control postoperatorio de las pacientes se efectúa a los 15 y 30 días para observar la cicatrización, además se toma citología de control a las 12 semanas postcono, sin haberse encontrado hasta el momento, alteración en alguno de los controles citológicos.

Una paciente presentó NIC III y se le efectuó el cono cursando un embarazo de 5 semanas, sin haberse efectuado el diagnóstico de éste, sino en los controles posteriores. Actualmente se encuentran dos pacientes más con embarazo posterior al cono, sin presentarse alteración en su curso y con control citológico y colposcópico normales.

\section{Discusión}

En el manejo actual de la Neoplasia Intraepitelial de Cervix, se ha demostrado la importancia de evaluar a la paciente que ha tenido una citología alterada con el colposcopio para tomar biopsias dirigidas de las lesiones, determinar la presencia de NIC, descartar lesión invasiva y la presencia o no de alteración glandular. Entre un 77-79\% la biopsia tomada bajo colposcopia para diagnóstico de NIC es igual al resultado histológico del cono y entre $14-21 \%$ el resultado del cono era mayor que el de la biopsia en 1 grado. (1-2).

En el tratamiento del NIC, los métodos de escisión (cono) a diferencia de los métodos destructivos (cauterio, crioterapia), permiten hacer el diagnóstico de invasión el cual no se ha diagnosticado en la colposcopia y/o en la biopsia y que se presenta en el 1-6\% de los conos; además 
permite también el diagnóstico de Adenocarcinoma Insitu, el cual no se diagnostica por colposcopia ni biopsia, en menos del $1 \%(3-8)$.

El diagnóstico del patólogo es definitivo en el tratamiento de las pacientes con patología cervical, pues disminuye la dependencia a la interpretación colposcópica. De allí la importancia de efectuar un procedimiento de escisión que bien puede ser con fines diagnósticos o de tratamiento (10).

La utilización de la radiocirugía en el campo de la ginecología está abriéndose paso cada día más. Dentro de las ventajas encontradas están: poder efectuar procedimientos a nivel del consultorio, requerir menos tiempo quirúrgico que el efectuado en salas de cirugía, utilización de anestesia local, ser ambulatoria, recuperación más rápida de la paciente, menor costo, pocas complicaciones inmediatas dentro de las que se encuentra la hemorragia la cual puede ser manejada rápidamente y la principal de todas no altera los bordes de sección, por lo tanto el patólogo puede hacer una evaluación más precisa de la pieza quirúrgica.

La radiocirugía saca el tejido más que destruirlo, lo cual ofrece mayor seguridad de escisión total de la lesión, aún en el canal, describiéndose sangramiento en menos del $2 \%$ y hemorragia secundaria en menos del $4 \%$. En análisis comparativos se ha observado la poca alteración que presenta la radiofrecuencia con «corte puro» en los bordes de sección del cono, siendo éste muy similar a lo observado al corte con bisturí $(9-13,21)$.

Los controles posteriores de las pacientes indican un postoperatorio mejor tolerado, escaso dolor, flujo vaginal y una cicatrización rápida sin alterar la zona de transformación ya que no se introduce ésta en el canal endocervical y permite así efectuar un mejor seguimiento citológico y colposcópico posterior.
Dentro de las alternativas en el manejo del NIC se encuentra al electrocauterio el cual tiene indicación en el tratamiento del NIC I y II pero se ha dejado de usar en el NIC III ya que requiere efectuar una cauterización profunda para abarcar toda la lesión y efectuar destrucción, sin poderse definir satisfactoriamente si se eliminó o no totalmente la lesión. Presenta mayor posibilidad de estenosis cervical y alteración de la zona de transformación lo que dificulta la observación posterior, aunque algunos autores reportan resultados excelentes con una rata de falla en el tratamiento de NIC III del 3 al $13 \%$ (14).

El uso de la crioterapia en el tratamiento del NIC ha dado muy buenos resultados con una rata de falla del $8 \%$ y un riesgo de recurrencia de lesión del $1 \%$ en el NIC I a 5 años, del $3 \%$ en el NIC II y de un 7 al $39 \%$ en el NIC III lo cual hace tener indicaciones muy precisas para su uso; además de producir un retroceso en la unión escamocolumnar hacia el canal cervical $(14-16,20)$.

Por último, efectuar el cono cervical «en frío», tiene los inconvenientes de ser de mayor costo, pues requiere evaluación preoperatoria, hospitalización, anestesia y uso de sala de cirugía; postoperatorio inmediato intrahospitalario, estenosis cervical o incompetencia de cervix (complicaciones en embarazos posteriores) y otros. Se describe la posibilidad de recurrencia del NIC III en 2-3\% y de Invasión en $0.9 \%(17-19)$.

\section{Conclusiones}

Se demuestra el uso de la radiocirugía como alternativa de tratamiento para aquellas pacientes con NIC que requieran cono cervical con fin de tratamiento o para pacientes que requieran cono con fines diagnósticos dadas las ventajas de costo, tiempo quirúrgico, resultados histológicos y seguimiento.

\section{BIBLIOGRAFIA}

1. Kirkup W., Singer AS. The accuracy of colposcopically directed biopsies in patients with suspected intraepithelial neoplasia of the cervix. Br. J. Obstet. Gynecol. 1980; 87: 1.

2. Veridiano NP., Delke I., Tanser L. Accuracy of colposcopically directed biopsy in patients with cervical neoplasia. Obstet. Gynecol. 1981; 58: 185 .

3. McIndoe GA et al. Laser excision rather than vaporization. The treatment of choise for cervical intraepithelial neoplasia. 1989; 74: 165.

4. Howe DT., Vicenti AC. Is large loop excision of the transformation zone more accurate than colposcopically directed punch biopsies in the diagnosis of cervical intraepithelial neoplasia? Br. J. Obstet. Gynecol. 1991; 98: 588.

5. Byrne PF., Santa Cassia LJ. Occult cervical carcinoma revealed by large loop diathermy. Lancet 1989; 2: 807.

6. Carter PG., Grant-Harris V., Wilson POG. Reliability of colposcopy and directed punch biopsy. Br. J. Obstet. Gynecol. 1991; 98: 232.

7. Mor-Yosef., Lopes A., Pearson S. Loop diathermy cone biopsy. Obstet. Gynecol. 1990; 75: 884.

8. Luesley DM et al. Loop diathermy excision of the cervical transformation zone in patients with abnormal cervical smear. $\mathrm{Br}$. Med. J. 1990; 300: 1690 .

9. Turner RJ. Tissue margins of cone biopsy specimens with «cold knife», CO2 \& Nd.YAG Lasers \& radiosurgery compared. Ob/Gin Radiosurgery Times 1991; 1: 1 .

10. Townsend DE., Kauffman K. Loop excision. Changing Colposcopy. OBG Management, June 1991.
11. OBG. News June 15-301991.

12. Prendiville W., Cullimore J., Normal S. Large loop excision of the transformation zone (L.L.E.T.Z.). Br. J. Obstet. Gynecol. 1989; 96 : 1054.

13. Anderson MC., Jones III, HW. ¿Debe la conización con «asa caliente» o rayos láser reemplazar a la biopsia cervical?. El Hospital 1992; $48: 4,8$.

14. Chanen W., Rome RM. Electrocoagulation diathermy for cervical dysplasia and carcinoma in situ. Year survey. Obstet. Gynecol. 1983; 61: 673 .

15. Richart RM. An analysis of «long term» follow up results in patients with cervical intraepithelial neoplasia treated by cryosurgery. Am. J. Obstet. Gynecol. 1980; 137: 823.

16. Townsend DE., Richart RM. Cryotherapy and carbon dioxide laser management of cervical intraepithelial neoplasia: a controlled comparison. Obstet. Gynecol. 1976; 48: 125.

17. Kolstad P., Lem V. Long-Term follow up of 1121 cases of carcinoma in situ. Obstet. Gynecol. 1976; 48: 125.

18. Piver MS. Manual of Gynecologic Oncology and Gynecology Little. Brown and Company. Firts edition 1989.

19. Disaia PJ., Creasman WT. Clinical Ginecologic Oncology 3 edition C.V. Mosby Company 1989.

20. Sevin BU. Invasive cancer of the cervix after cryosurgery pitfalls of conservative management. Obstet Gynecol 1979; 53: 465.

21. Turner RJ et al. Analysis of tissue margins of cone biopsy specimens obtained with "cold Knife», $\mathrm{CO} 2$ and Nd.YAG lasers and a radiofrecuency surgical unit. J. Reprod. Med. 1992; 37(7): 607. 